\title{
Sleep improvement after hip arthroplasty: a study on short-stem prosthesis
}

\author{
Josef Hochreiter ${ }^{1} \cdot$ Harald Kindermann ${ }^{2} \cdot$ Mattiassich Georg $^{3} \cdot$ Reinhold Ortmaier $^{1,4} \cdot$ Marian Mitterer $^{5}$ (D)
}

Received: 17 March 2019 / Accepted: 8 July 2019 / Published online: 27 July 2019

(C) The Author(s) 2019, corrected publication August 2019

\begin{abstract}
Purpose The purpose of this study was to evaluate sleep disturbance prospectively before and after short-stem hip arthroplasty. Methods A prospective study on 25 patients undergoing a primary unilateral total short-stem hip replacement was conducted. Patients were observed for six months. To evaluate the sleep quality and daytime sleepiness, the Pittsburgh Sleep Quality Index and Epworth Sleepiness Scale were used. To assess the general physical health status, we used the Short Form 36 Health Survey (SF-36). Pain was recorded on a visual analog scale.

Results The physical health status of the patients improved significantly $(p<0.05)$ during the six month follow-up period in seven out of nine categories. During the first post-operative week, the sleep quality stayed on an equal level to the pre-operative state, following a steady improvement over the next months $(6$ months $p=0.00)$. The daytime sleepiness showed a significant improvement during all the follow-ups $(6$ months $p=0.00)$. Pain decreased significantly from baseline to six months postoperatively $(p=0.00)$. There was no correlation between pain and sleep quality or pain and daytime sleepiness.

Conclusion According to our results, patients undergoing short-stem total hip arthroplasty can expect a 50\% improvement of sleep quality and physical function six months after surgery.
\end{abstract}

Keywords Total hip arthroplasty $\cdot$ Short-stem hip arthroplasty $\cdot$ Sleep $\cdot$ Pain $\cdot$ Hip osteoarthritis

\section{Introduction}

Hip osteoarthrosis is a common condition, and approximately one-third of adults in the USA and up to $23 \%$ of the adults in Europe are affected [1,2]. Women have

The original version of this article was revised: Authors first and last names have been interchanged.

Hochreiter Josef and Mitterer Marian contributed equally to this work.

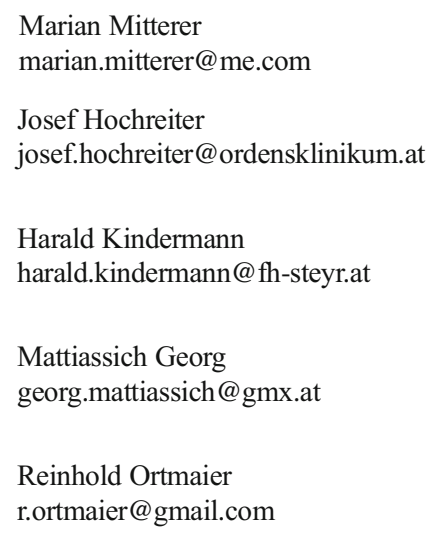

a higher incidence of hip osteoarthrosis than men, at a ratio of $2: 1$ [3].

According to the World Health Organization, $80 \%$ of adults with osteoarthrosis have limitations in movement, and $25 \%$ cannot perform their major daily activities of life [3]. Patients
1 Department of Orthopedic Surgery, Ordensklinikum Barmherzige Schwestern Linz, Vinzenzgruppe Center of Orthopedic Excellence, Teaching Hospital of the Paracelsus Medical University, Salzburg, Austria

2 Department of Marketing and Electronic Business, University of Applied Sciences Upper Austria, Campus Steyr, Wels, Austria

3 Trauma Center Graz, Graz, Austria

4 Institute for Sports Medicine, Alpine Medicine and Health Tourism (ISAG), Tirol Kliniken GmbH, Innsbruck and UMIT, Hall in Tirol, Austria

5 Department of Orthopaedics and Traumatology, Paracelsus Medical University Salzburg, Müllner Hauptstrasse 48, 5020 Salzburg, Austria 
with hip osteoarthrosis often describe a reduced social function as well as worse sleep quality and an increase in sleep medication use [4-6].

A reduced sleep quality correlates with increased night pain and severity of osteoarthrosis [7].

The current gold standard for hip osteoarthrosis is a total hip replacement [8]. For younger patients with hip osteoarthrosis, the short-stem hip prosthesis is a new method that promises reduced surgical trauma as well as faster post-operative recovery compared with standard-stem prosthesis $[9,10]$.

After total hip replacement, studies have shown a distinct increase in quality of life and energy/vitality (SF-36) as well as reduced daily and night pain [11-13].

Previous studies observed sleep quality, pain, and physical function in patients undergoing hip and knee arthroplasty up to 24 months after surgery $[11,12,14]$. Their results found improved sleep quality and decreased pain after arthroplasty but with an initial worsening and weeks of delay [15].

Prospective studies are lacking, and no information exists about sleep disturbance after short-stem hip arthroplasty.

This study intended to determine the sleep quality and daytime sleepiness in a prospective evaluation during several defined follow-up time points (up to 6 months post-operative) to identify correlations and to improve the preoperative information giving to patients.

\section{Materials and methods}

A prospective cohort study was conducted on patients with primary unilateral hip arthroplasty from January 2016 to August 2016.

We included patients undergoing primary unilateral shortstem hip arthroplasty. The mean age of the study collective was 68.0 years. The indication for surgery was primary osteoarthritis on the affected hip. The inclusion criteria were patients older than 18 years who agreed to and were able to complete the pre- and post-operative surveys.

The exclusion criteria were patients who presented any other indication than primary osteoarthritis such as revision surgery, patients with infectious complications after surgery, history of pre-existing sleep disorder, history of supplemental sleeping aids and patients with dementia or mild cognitive impairment as well as patients with sleep apnea syndrome.

Table 1 Demographics (age, sex, BMI) of the study population

Patients $(n=25)$

\begin{tabular}{ll}
\hline Age (year) & $68.0 \pm 9.7$ \\
Body mass index $\left(\mathrm{kg} / \mathrm{m}^{2}\right)$ & $26.5 \pm 5.0$ \\
Sex female & $19(76.0 \%)$ \\
\hline
\end{tabular}

Overall, 25 patients were included. The details of the patient demographics are shown in Table 1. In all the patients, the PSQI, Epworth Sleepiness Scale, SF-36, and VAS were obtained pre-operatively, one week, three weeks, six weeks, three months, and six months post-operatively.

Ethical approval was obtained, and all the patients signed an informed consent before participating in this study.

Patients received no supplemental sleeping aids during the study period and no prescription afterward. The average hospital stay was six \pm two (range, 3-8) days.

The current health status was evaluated using the Short Form 36 Health Survey (SF-36) [16]. This questionnaire measured eight multi-item variables using 36 self-rating questions. For each variable, the item scores were coded, summed, and transformed onto a scale from 0 (worst possible health status) to 100 (best possible health status) [16].

All the 25 patients had to fill out the SF-36 pre-operatively and six months after the surgery.

For assessing the sleep disturbance and quality, we used both the Pittsburgh Sleep Quality Index (PSQI) and the Epworth Sleepiness Scale (ESS).

The PSQI is a self-rated questionnaire that collects nineteen individual items to measure sleep quality and disturbances. The seven categories were subjective sleep quality, sleep latency, sleep duration, habitual sleep efficiency, sleep disturbances, use of sleeping medication, and daytime dysfunction. These categories were summed up to create a total score with a maximum of 21. A higher score indicates worse sleep dysfunction, and a score greater than five indicates poor sleep quality. All the study patients were asked to fill out the PSQI pre-operatively and during the post-operative checkups in the following intervals: one week, three weeks, six weeks, three months, and six months. Patients who could not attend every checkup were called and completed the survey over the telephone.

To assess the daytime sleepiness, we used the ESS. The ESS is a validated questionnaire that quantifies the severity of daytime sleepiness [17]. An ESS score higher than ten indicates significant sleepiness. The ESS questionnaire was conducted preoperatively as well as postoperatively after one week, three weeks, six weeks, three months, and six months.

To assess the pain pre-operatively and after the surgery, we used a visual analog scale (VAS) from 0 to 10 .

Furthermore, demographic data such as sex, age, and body mass index were collected to analyze subsidiary factors to sleep disturbance.

\section{Statistical analysis}

Statistical analysis was performed using SPSS for Windows, version 25 . The categorical variables are expressed as frequency and percentage, whereas ordinal variables are represented as the median and interquartile range (25th percentile; 75th percentile). The Wilcoxon signed-rank test was used to compare 
the nonparametric time-dependent variables. Differences were considered statistically significant if $p<0.05$.

\section{Surgical technique}

For all the patients, an anterolateral, modified Watson-Jones approach was used. A cement-less, monobloc short-stem (Optimys; Mathys, Bettlach, Switzerland) and a cement-less press-fit, monobloc vitamin E-enriched HXLPE cup coated with titanium particles (RM Pressfit vitamys; Mathys, Bettlach, Switzerland) were used. All the patients received full weight-bearing ambulation under surveillance of physiotherapy and started using crutches immediately on post-operative day 1 with an initial restriction of flexion at $90^{\circ}$ for one week.

\section{Rehabilitation}

Full weight-bearing using crutches under surveillance of a physiotherapist was allowed immediately on post-operative day 1. Functional active and passive motion, with initial restriction of $90^{\circ}$ of flexion for one week was allowed. After four to six weeks, all patients underwent inpatient or outpatient rehabilitation program five days a week for two to three weeks.

\section{Results}

A significant improvement could be detected in seven out of the nine subcategories of the SF-36. Only the subcategories Social Function and Health Perceptions showed nonsignificant results due to missing figures.

The pre-operative mean physical function was $38.3 \% \pm$ $24.1 \%$, and the energy/vitality was $47.4 \% \pm 18.7 \%$ in adults with unilateral hip osteoarthrosis. During the following six months, the post-operative physical function improved to $61.8 \% \pm 1.5 \%(p=0.00)$, and the energy/vitality increased to $60.4 \% \pm 15.6 \%$. A Wilcoxon signed-rank test revealed that this improvement was significant, $z=-4.29, p=0.00$, with a large effect size $(r=0.88)$ (Table 2).

The average pre-operative PSQI score was $9.1 \pm 3.9$ in the 25 participating patients. The PSQI score increased slightly over the first post-operative week following a nearly uninterrupted decrease over the next weeks and months. Six months after surgery, the mean PSQI was $4.3 \pm 2.0(z=-3.22, p=$ $0.00, r=0.80$ ), which indicates a normal sleep (Fig. 1).

The greatest improvements from the pre-operative period until six months after surgery were made in the categories of subjective sleep quality, sleep latency, sleep duration, habitual sleep efficiency, and daytime dysfunction.

The ESS dropped significantly from a pre-operative value of $7.4 \pm 5.6$ to $4.2 \pm 3.6(z=-3.61, p=0.00, r=0.72)$ during the following weeks. The improvement of daytime sleepiness was comparable with the PSQI results.
VAS score decreased continuously from baseline $5.5 \pm 1.5$ over all the points and checkups during the six months postoperative follow-up until $0.1 \pm 0.3(z=-4.43, p=0.00, r=0.89)$.

There was no significant correlation between the VAS and PSQI and between the VAS and Epworth sleepiness scale.

\section{Discussion}

Sleep disorder in patients with osteoarthrosis in the hip or other joints is a common condition [18]. Pain is an essential parameter in patients with sleep disturbance and osteoarthrosis $[8,18,19]$. Multiple studies have demonstrated that primary hip arthroplasty improves patients' quality of life as well as sleep disturbance after surgery. Most of the previous studies focused on the hospitalization period and the first weeks after surgery (up to 3 months) $[8,18,20]$.

This study was conducted to observe changes in sleep disorder in patients with unilateral hip osteoarthrosis undergoing a primary short-stem hip replacement. The goal was to collect patient data from a pre-operative baseline period until reintegration to normal daily activities six months after surgery.

The patients reported poor sleep quality at the beginning of the study, which is comparable with other studies that showed increased pain and lack of sleep in patients with hip osteoarthrosis [7, 11].

As shown in other studies, sleep quality decreases in the first week after surgery to increase again in the following weeks to months [15]. Other studies have already established poor sleep quality at baseline levels up to one month after surgery in patients with total hip replacement [21]. In our study, sleep quality according to PSQI stayed the same level at the first week after surgery following a substantial improvement in all categories, except sleep medication abuse, over the following weeks. Even three months after surgery, there was still improvement relating to PSQI. The Epworth sleepiness scale, which investigated the daytime sleepiness as well as the VAS pain scores, improved substantially after the first postoperative week. The Epworth sleepiness scale did not change significantly after the first post-operative week, while the VAS showed a continuous drop after surgery and was never above the baseline level during the study [22].

Regarding the quality of life, our study suggests a significant improvement after hip arthroplasty in patients with osteoarthrosis. Pre-operatively, the participants had poor SF36 scores in physical functioning, general health, and body pain. This correlates to pre-existing studies suggesting reduced health and quality of life in patients with chronic osteoarthrosis [12]. After surgery, the greatest improvements were in the scores for physical functioning, role physical, general health, and body pain $[11,14]$. Moreover, improvements in social function, vitality, and mental health were observed. These findings suggest that improved physical function and general 
Table 2 Change in SF 36, PSQI, ESS, and VAS after short-stem hip arthroplasty during the study at the 6 checkups

\begin{tabular}{|c|c|c|c|c|c|c|}
\hline & Pre-operative & 1 week & 3 weeks & 6 weeks & 3 months & 6 months \\
\hline \multicolumn{7}{|l|}{ SF-36 } \\
\hline Physical functioning & $38.3 \%$ & - & - & - & - & $61.8 \%$ \\
\hline Role physical & $25.0 \%$ & - & - & - & - & $60.0 \%$ \\
\hline Pain & $29.0 \%$ & - & - & - & - & $84.5 \%$ \\
\hline Change in health & $31.0 \%$ & - & - & - & - & $72.0 \%$ \\
\hline Energy/vitality & $47.4 \%$ & - & - & - & - & $60.4 \%$ \\
\hline Social functioning & $72.5 \%$ & - & - & - & - & $80.5 \%$ \\
\hline Role emotional & $65.2 \%$ & - & - & - & - & $89.4 \%$ \\
\hline Mental health & $73.9 \%$ & - & - & - & - & $77.9 \%$ \\
\hline Health perceptions & $65.0 \%$ & - & - & - & - & $63.0 \%$ \\
\hline PSQI & $9.6 \pm 3.9$ & $9.5 \pm 4.4$ & $6.3 \pm 2.9$ & $5.4 \pm 2.8$ & $5.8 \pm 2.8$ & $4.3 \pm 2.0$ \\
\hline Duration of sleep & $1.0 \pm 1.1$ & $1.0 \pm 1.2$ & $0.6 \pm 1.0$ & $0.4 \pm 0.7$ & $0.7 \pm 0.9$ & $0.0 \pm 0.0$ \\
\hline Sleep disturbance & $1.7 \pm 0.5$ & $1.6 \pm 0.5$ & $1.3 \pm 0.5$ & $1.2 \pm 0.4$ & $1.2 \pm 0.4$ & $1.2 \pm 0.4$ \\
\hline Sleep latency & $1.8 \pm 0.8$ & $1.8 \pm 0.8$ & $1.3 \pm 1.0$ & $1.3 \pm 1.1$ & $1.3 \pm 0.9$ & $1.1 \pm 1.0$ \\
\hline Day dysfunction & $1.1 \pm 0.6$ & $1.0 \pm 0.9$ & $0.7 \pm 0.7$ & $0.4 \pm 0.6$ & $0.6 \pm 0.7$ & $0.5 \pm 0.7$ \\
\hline Sleep efficiency & $1.5 \pm 1.2$ & $1.5 \pm 1.2$ & $0.8 \pm 0.8$ & $0.7 \pm 0.9$ & $0.8 \pm 1.1$ & $0.3 \pm 0.6$ \\
\hline Sleep quality & $1.4 \pm 0.7$ & $1.4 \pm 0.8$ & $1.1 \pm 0.5$ & $0.9 \pm 0.5$ & $0.7 \pm 0.6$ & $0.8 \pm 0.6$ \\
\hline Medication & $0.7 \pm 1.2$ & $1.0 \pm 1.4$ & $0.7 \pm 1.2$ & $0.8 \pm 1.3$ & $0.7 \pm 1.2$ & $0.7 \pm 1.2$ \\
\hline ESS & $7.4 \pm 5.6$ & $5.8 \pm 4.8$ & $4.3 \pm 2.6$ & $4.5 \pm 2.8$ & $3.9 \pm 2.8$ & $4.2 \pm 3.6$ \\
\hline VAS pain & $5.5 \pm 1.5$ & $3.6 \pm 1.7$ & $2.1 \pm 1.8$ & $0.8 \pm 0.9$ & $0.2 \pm 0.5$ & $0.1 \pm 0.3$ \\
\hline
\end{tabular}

health after surgery in patients with chronic osteoarthrosis have a mild impact on nonphysical related limitations [12, 23].

The limitations of this study include a small cohort. A relatively unequal spreading in gender, the female to male ratio was $76 \%: 24 \%$, the preliminary exclusion criteria of patients with revision hip arthroplasty and patients using sleeping medication. Another limitation of this study is the absence of a control group, especially of a standard-stem group, which permits to draw definitive conclusions about differences in sleep recovery between standard and short stems.

However, the difference in the quality of life after shortstem and standard-stem hip arthroplasty has already been reviewed in several studies that suggest similar functional outcome with slight benefit for short stem prosthesis in younger patients due to faster post-operative recovery as well as less stress shielding [9, 10, 24, 25]. A supplement reduction of thigh pain using short-stem hip arthroplasty might has an
Fig. 1 Pre-operative and postoperative PSQI, ESS, and VAS after short-stem hip arthroplasty. Pre-operative and post-operative PSQI, ESS, and VAS during the period of record. The diagram shows a clear trend of increase in sleep quality (PSQI), as well as a decrease in daytime sleepiness (ESS) and pain (VAS) compared with baseline

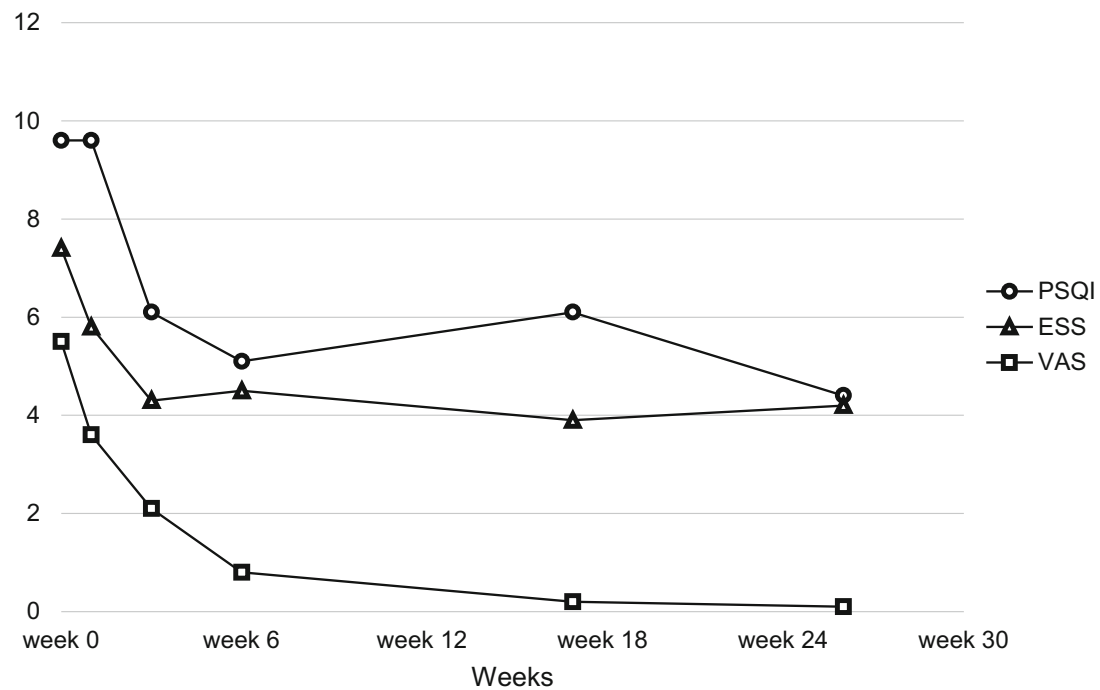


effect on sleep improvement, but existing studies show no significant difference compared with conventional standardstem hip arthroplasty [26].

Nevertheless, a prospective, randomized control trial including two groups of short stems and standard stems would help to show if there are real differences of sleep recovery between standard- and short-stem hip arthroplasty.

This study demonstrates a post-operative decrease or steady state of sleep quality in patients with unilateral short-stem hip arthroplasty. However, in the following weeks and months, patients can expect substantial improvement regarding sleep quality, daytime sleepiness, quality of life, and pain. This information can help to shape patients' expectations pre-operatively and reducing direct post-operative frustration due to potential sleep improvement even three months after surgery.

Funding Information Open access funding provided by Paracelsus Medical University.

Compliance with ethical standards Ethical approval was obtained, and all the patients signed an informed consent before participating in this study.

Conflict of interest The authors declare that they have no conflict of interest.

Open Access This article is distributed under the terms of the Creative Commons Attribution 4.0 International License (http:// creativecommons.org/licenses/by/4.0/), which permits unrestricted use, distribution, and reproduction in any medium, provided you give appropriate credit to the original author(s) and the source, provide a link to the Creative Commons license, and indicate if changes were made.

\section{References}

1. Lawrence RC, Felson DT, Helmick CG (2008) Estimates of the prevalence of arthritis and other rheumatic conditions in the United States. Part II. Arthritis Rheum 58(1):26

2. Schaap L (2001) European Project on Osteoarthritis (EPOSA): methodological challenges in harmonization of existing data from five European population-based cohorts on aging. BMC Musculoskelet Discord 12:272

3. World health organization Chronic diseases and health promotion (2019) Chronic rheumatic conditions https://www.who.int/chp/ topics/rheumatic/en/. Accessed 15 January 2019

4. Edwards MH, van der Pas S, Denkinger MD, Parsons C, Jameson KA, Schaap L (2014) Relationships between physical performance and knee and hip osteoarthritis: findings from the European Project on Osteoarthritis (EPOSA). Age Ageing 43(6):806-813

5. Parmelee PA, Tighe CA, Dautovich ND (2015) Sleep disturbance in osteoarthritis: linkages with pain, disability and depressive symptoms. Arthritis Care Res 67(3):358

6. McCurry SM, Von Korff M, Vitiello MV (2011) Frequency of comorbid insomnia, pain, and depression in older adults with osteoarthritis: predictors of enrollment in a randomized treatment trial. J Psychosom Res 71(5):296

7. Woolhead G, Gooberman-Hill R, Dieppe P (2010) Night pain in hip and knee osteoarthritis: a focus group study. Arthritis Care Res 62(7):944
8. Fielden JM, Gander PH, Horne JG, Lewer BM, Green RM, Devane PA (2003) An assessment of sleep disturbance in patients before and after total hip arthroplasty. J Arthroplast 18(3):371-376

9. Henry BM, Wražeń W, Pąchalska M (2016) Health-related qualityof-life and functional outcomes in short-stem versus standard-stem total hip arthroplasty: an 18-month follow-up cohort study. Med Sci Monit 22:4406-4414

10. Van Oldenrijk J, Scholtes VAB (2017) Better early functional outcome after short stem total hip arthroplasty? A prospective blinded randomised controlled multicentre trial comparing the Collum Femoris Preserving stem with a Zweymuller straight cementless stem total hip replacement for the treatment of primary osteoarthritis of the hip. BMJ Open

11. Rissanen P, Aro S, Sintonen H (1996) Quality of life and functional ability in hip and knee replacements: a prospective study. Qual Life Res 5(1):56

12. March LM, Cross MJ, Lapsley H (1999) Outcomes after hip or knee replacement surgery for osteoarthritis. A prospective cohort study comparing patients' quality of life before and after surgery with age-related population norms. Med J Aust 171(5):235

13. von Rottkay E, Rackwitz L, Rudert M, Nöth U, Reichert JC (2018) Function and activity after minimally invasive total hiparthroplasty compared to a healthy population. Int Orthop 297-302

14. Rissanen P, Aro S, Slatis P (1995) Health and quality of life before and after hip or knee arthroplasty. J Arthroplast 10(2):169

15. Krenk L, Jennum P, Kehlet H (2013) Activity, sleep and cognition after fast-track hip or knee arthroplasty. J Arthroplast 28(8):1265

16. Jenkinson C, Coulter A, Wright A (1993) Short form 36 (SF36) health survey questionaire: normative data for adults of working age. BMJ 306:1437-1440

17. Johns MW (1991) A new method for measuring daytime sleepiness: the Epworth sleepiness scale. Sleep 14(6):540

18. Manning BT, Kearns SM, Bohl DD (2017) Prospective assessment of sleep quality before and after primary total joint replacement. Orthopaedics 40(4):636-640

19. Foley D, Ancoli-Israel S, Britz P (2004) Sleep disturbances and chronic disease in older adults: results of the 2003 National Sleep Foundation Sleep in America Survey. J Psychosom Res 56(5):497

20. Krenk L, Jennum PH (2012) Sleep disturbances after fast-track hip and knee arthroplasty. Br J Anaesth 109(5):769-775

21. Buysse DJ, Reynolds CF III, Monk TH (1989) The Pittsburgh sleep quality index: a new instrument for psychiatric practice and research. Psychiatry Res 28(2):193

22. Wylde V, Rooker J, Halliday L (2011) Acute postoperative pain at rest after hip and knee arthroplasty: severity, sensory qualities and impact on sleep. Orthop Traumatol Surg Res 97(2):139

23. Myoji Y, Fujita K, Mawatari M (2014) Changes in sleep-wake rhythms, subjective sleep quality and pain among patients undergoing total hip arthroplasty. Int J Nurs Pract 21(6):764-770

24. Merschin D, Häne R, Tohidnezhad M, Pufe T, Drescher W (2018) Bone-preserving total hip arthroplasty in avascular necrosis of the hip-a matched-pairs analysis. Int Orthop:1509-1516

25. Miladi M, Villain B, Mebtouche N, Bégué T, Aurégan JC (2018) Interest of short implants in hip arthroplasty for osteonecrosis of the femoral head: comparative study "uncemented short" vs "cemented conventional” femoral stems. Int Orthop 42(7):1669-1674. https:// doi.org/10.1007/s00264-018-3981-0

26. Pogliacomi F, Schiavi P, Grappiolo G, Ceccarelli F, Vaienti E (2019) Outcome of short versus conventional stem for total hip arthroplasty in the femur with a high cortical index: a five year follow-up prospective multicentre comperative study. Int Orthop

Publisher's note Springer Nature remains neutral with regard to jurisdictional claims in published maps and institutional affiliations. 